\title{
Comparison of optic disc image assessment methods when examining serial photographs for glaucomatous progression
}

Chris J Barry, Robert Eikelboom, Yogesan Kanagasingam, Ludmila Jitskaia, William Morgan, Phillip House, Max Cuypers

\begin{abstract}
Aim-To assess serial, simultaneous stereo optic disc images by four methods for glaucomatous progression.

Methods-Using varying techniques, two ophthalmologists assessed serial optic disc images of 52 eyes from 27 patients with a mean duration between images of 18 months. The neuroretinal rim width was qualitatively assessed by four assessment methods and compared with quantitative rim measurements made using PC based software.

Results-The highest sensitivity of $83 \%$ was achieved using computerised stereo chronoscopy.

Conclusion-Stereo chronoscopy improved the detection of subtle optic disc changes when compared with simpler assessment techniques.

(Br f Ophthalmol 2000;84:28-30)
\end{abstract}

Observation of the optic disc is a key factor in the management of glaucoma and can be aided by using serial optic disc photographs. Stereo photography and stereoscopic viewing give an enhanced perception of disc topography assisting the interpretation of small changes in the disc structure. Stereo chronoscopy is a tool that can be used to view serial stereo pairs of images. The images need to be aligned so that they are superimposed and viewed alternately. Any changes which have occurred between imaging sessions appear to "flutter" while the rest of the image is stable when the serial images are flickered. Stereo chronoscopy has been shown to improve the sensitivity of detection of small disc changes ${ }^{1-4}$ but has not gained acceptance owing to stereo base fluctuations from sequential stereo imaging.

Simultaneous stereo photographs, in which one frame records both stereo views at the same moment in time, eliminates or reduces variations in the stereo base. We have previously described an optical/mechanical device and personal computer (PC) based methods of stereo chronoscopy to view simultaneous stereo images. ${ }^{5}{ }^{6}$ In this study, the ability to detect changes in neuroretinal rim width from simultaneous stereo images was assessed using stereo chronoscopy and two simpler comparative methods. The stereo chronoscopy software also included the facility for manual neuroretinal rim measurements (stereo chronometry) and was used as the gold standard.
Method

Two ophthalmologists compared the serial simultaneous stereo optic disc images of 52 eyes from 27 patients by four methods (time between images 18 months, range 9-27 months).

Pupils were dilated and subjects were assessed at the slit lamp using a hand held 60D lens to observe the optic disc. Photographs taken at a previous attendance were inserted into a half frame stereo viewer (3d Reel Enterprises, USA) adjacent to the slit lamp eyepieces.

The serial slides were alternatively loaded into a half frame stereo viewer and the slides assessed for any change.

An optical, stereo flicker comparator was designed and built based on the Pulfrich model. ${ }^{7}$ Figure 1 is a schematic diagram of the comparator. The comparator was placed over a light box and two photographic slides placed in the slide stage. Movement of a shutter allowed each slide to be viewed alternately. The right slide holder could be moved in the horizontal plane to align the right slide with the left slide. Both slide light paths are brought to a common path via a two way mirror and viewed with an attached commercial half frame stereo viewer. The stereo viewer was

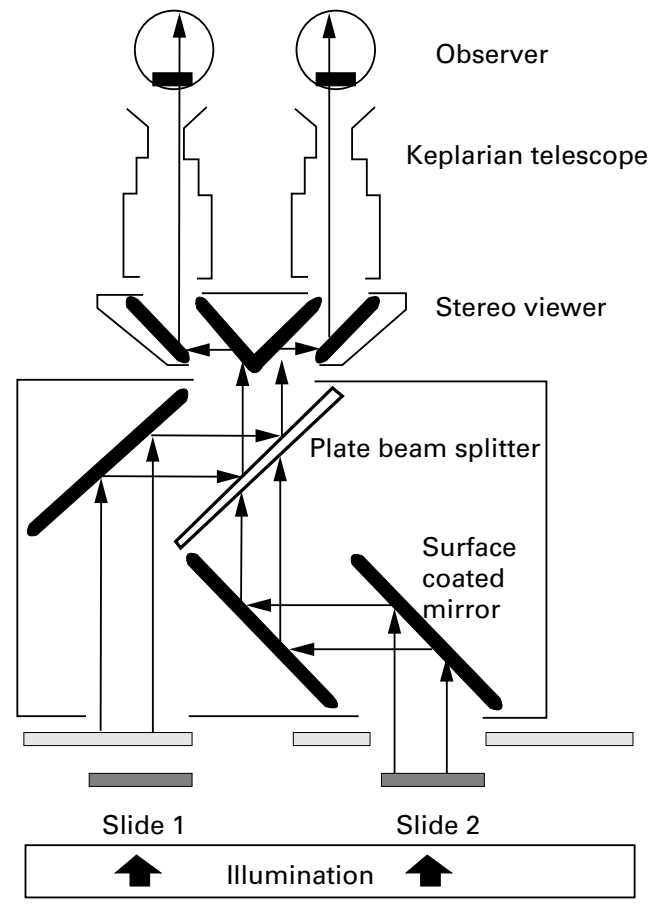

Figure 1 Schematic diagram of the optical/mechanical stereo flicker chronoscopy comparator. 
adapted with rotating prisms to allow an adjustment for differing interpupillary distances. The eyepieces were replaced by telescopic viewing devices which increased the magnification and were independently adjustable for optimal focusing.

The photographic optic disc slides were scanned and digitised using a Polaroid SprintScan 35 (Cambridge, MA, USA) in 24 bit colour. Software was developed to align the images so that the images could be superimposed. The program then generated an interlaced image from the stereo pair to produce one single image. The observer wore $3 \mathrm{D}$ stereo goggles which contain liquid crystal display (LCD) shutters (3DMax, Kasan Electronics, Anyang, Korea) synchronised to the monitor. ${ }^{6}$ The observer saw the left image of the stereo pair with the left eye and right image with the right eye forming a stereo view. Two images were presented in rapid sequence on the monitor, with switching controlled from the keyboard by the user.

The computer stereo software also incorporated the facility for manual, stereo, neuroretinal rim measurement (stereo chronometry). An electronic, predefined template marked with 18 points was placed over the image as a guide for measurements; the template was transferred to the second serial image maintaining the alignment. The vertical disc height was measured for each disc and a magnification factor used where appropriate. Using the mouse pointer, the operator identified the inner and outer neuroretinal rim margins at each of the 18 measurement points. The outer rim was defined as the junction between neural tissue and the scleral ring. The inner rim margin was defined as the junction between the disc floor and upward slope of the rim. The measurements were repeated a further four times.

The five radial measurements from each point were compared from the earliest image to the most recent using a two tailed Student's $t$ test (Sigma Stat Jandell Scientific, San Rafael, CA, USA). A conservative judgment for rim change was determined empirically as two adjacent points where the probability $(p)$ of differences between two sets of five measurements was $<0.001$. Sensitivity and specificity were calculated for each observer for the four qualitative methods using the rim measurement as a gold standard.

\section{Results}

Six eyes from the 52 were found to have significant rim width changes. Table 1 summarises the sensitivity and specificity of each of the four methods for the two observers. As only six rims changed from the 52 eyes studied

Table 1 Sensitivity and specificity of the two observers' assessments compared with the gold standard, expressed as percentages

\begin{tabular}{|c|c|c|c|c|}
\hline \multirow[b]{2}{*}{ Method } & \multicolumn{2}{|l|}{ Observer 1} & \multicolumn{2}{|l|}{ Observer 2} \\
\hline & Sensitivity & Specificity & Sensitivity & Specificity \\
\hline Stereo viewer at slit lamp eyepieces & 33 & 90 & 33 & 85 \\
\hline Stereo viewer & 50 & 97 & 33 & 92 \\
\hline Optical/mechanical comparison & 50 & 87 & 67 & 87 \\
\hline Computerised chronoscopy & 83 & 92 & 83 & 87 \\
\hline
\end{tabular}

the specificity was high for all four methods. The table also shows that the sensitivity varied between the two observers and the qualitative method used. There was a progressive improvement of sensitivity from methods one to four. The computerised chronoscopy had the highest sensitivity of $83 \%$ for both observers.

\section{Discussion}

Most previous planimetric disc analyses have relied on coefficient of variance (CV) for statistical comparisons (standard deviation/ mean $\times 100$ ). Glaucomatous progression is associated with either concentric or focal decreases in rim area. With the measurement mean as the denominator, the $\mathrm{CV}$ is likely to increase with smaller rim areas or vary due to physiological variations in disc size. The two tailed Student's $t$ test was used to analyse our multiple radial rim measurements so that a probability of change for each individual rim point could be calculated and thus focal rim narrowing could be more readily detected. As both series of measurements were taken at the same session by a single observer, both interobserver and intersessional variations were minimised.

The optical/mechanical comparator was found to be both quick and simple to use. In general, poor quality images or images with noticeable colour changes did not impair disc assessment. The technique of computerised flicker chronoscopy was considered the method with the highest potential and worthy of further improvement, particularly when coupled with the measurement software. The disadvantage of the computer methods was the time taken to complete the scanning, alignment, magnification, and assessment components. It was noted that the images displayed on the computer monitor did not appear to have the same crispness and clarity when compared with the photographic slides viewed in the optical/mechanical comparator.

\section{Conclusion}

Changes in the appearance of the optic disc are still considered a key factor in the assessment of glaucoma. This project assessed stereo flicker chronoscopy to examine simultaneous stereo, serial optic disc images for neuroretinal rim narrowing and was found to increase the detection of rim change compared with simpler methods. The long term validity and reproducibility for new glaucoma imaging technologies has yet to be determined. Until newer technologies displace optic disc imaging, the improvement of existing techniques to recognise small structural changes within the optic disc remains relevant.

1 Goldmann H, Lotmar W. Quantitative studies in stereochronoscopy: application to the disc in glaucoma I. Phenomenology. Graefes Arch Clin Exp Ophthalmol 1964;222:38-42.

2 Bengtsson B, Krakau CET. Flicker comparison of fundus photographs: a technical note. Acta Ophthalmol (Copenh) photographs:

3 Heiil A, Bengtsson B. Diagnosis of early glaucoma with flicker comparisons of serial disc photographs. Invest Ophthalmol Vis Sci 1989;30:2376-84. 
4 Takamoto T, Schwartz B. Stereochronometry: quantitative measurement of optic disc cup changes. Invest Ophthalmol Vis Sci 1985;26:1445-9.

5 Barry CJ, Kanagasingam Y, Jitskaia L, et al. A personal computer based method of stereo chronometry for measuring neuroretinal rim width: a pilot study. Aust NZ $\mathcal{f}$ Ophthalmol 1998;26:(suppl)S22-5.
6 Kanagasingam Y, Barry CJ, Jitskaia L, et al. Software for 3-D visualization/analysis of optic disc images. IEEE Engineering in Biology and Medicine Magazine 1999;18:43-9.

7 Pulfrich C Über die nutzbarmachung des stereokomparator für den monocularen gebrauch and über ein hierfur bestimmtes monokulares verleichs-microskop. Zeitschrift Instrumenten kunde 1904:161. 